\title{
Application of Designed Orientation Program for Nurse Interns Based on Learning Needs Assessment
}

Heba K. Obied, Assistant lecturer in Nursing Administration Dep, Faculty of Nursing, Tanta University

Fouada M.Shabaan, Prof. of Nursing Administration, Faculty of Nursing, Tanta University

Helmy H. Shalaby, Prof. of Plastic Surgery, Faculty of Medicine, Tanta University

Samar H. Gadiry, Lecturer of Nursing Administration, Faculty of Nursing, Tanta University

\begin{abstract}
Orientation programs based on LNA encourage nurse interns to feel safe and enable them to demonstrate competency in the basic clinical and management skills needed for providing high quality and safe patient care. Using LNA ensure NIs commitment to these program. This study aimed to design, implement, and evaluate an orientation program about clinical and management skills needed for nurse interns based on the findings of learning needs assessment tool. Material and method the study was conducted at Tanta University Faculty of Nursing and Tanta University Emergency and Main Hospitals ICUs and obstetric and dialysis departments. (384) nursing students passed the $4^{\text {th }}$ academic year attended the orientation program. (198) spent their internship year at Tanta University Hospitals were included in the researcher's observation 3 month post program. The data collection was achieved by using LNA scale, knowledge test, NIs' orientation program and follow up observation sheet. RESULTS: Pre program around half (51\%) of NIs assessed themselves as cannot carryout different clinical and management skills. Majority (87\%) of NIs got low knowledge level for both skills. Post orientation program statistical significant improvement at $(\mathrm{P} \leq 0.05)$ was found in NIs' knowledge and practical levels for both clinical and management skills. Conclusion designing orientation programs before starting the internship year based on nurse interns LNA is important to ensure efficient interns performance. Recommendation conduct orientation programs based on nurse interns' LNA and knowledge test before starting internship year and at the beginning of each new rotation, provide nurse interns with pass booklet include standard procedures required to be carried out in each rotation, provide them also with designed evaluation manual based on the pass booklet and implementing nursing mentor-ship and preceptor-ship programs during internship year.
\end{abstract}




\section{Introduction}

The internship year is considered a time of transition from undergraduate nursing students to beginning level registered nurses. During this year nurse interns should acquire the values, attitudes, and goals fundamental to the nursing profession $^{(1,2)}$. At the beginning of the internship year many nurse interns feel uncomfortable and inadequacy as their skill levels do not match their expectations of the role and responsibilities of a practicing as a registered nurse ${ }^{(3)}$. During internship year nurse interns are expected to work in intensive care units (ICUs) under supervision of experienced knowledgeable supervisors ${ }^{(4)}$.

Today's health care arena become complex and suffer from severe nursing shortage and limited budgets for continuing education programs; that affect organization's ability to provide comprehensive orientation programs for new graduates or for nurse interns ${ }^{(5-6)}$.

In addition hospital administrators' expect nurse interns to be competent to function and take responsibilities in nursing service at the time of graduation with adequate clinical and patient management skills to cover shortage and decrease the workload on experienced staff. Consequently nurse interns are imposed to the work field responsibilities early ${ }^{(7-8)}$. Therefore, nursing faculties have to provide interns with a supportive clinical environment in terms of skills, knowledge, practice, reflection and cultural socialization to help them to feel competent and supported ${ }^{(9)}$.

Effective internship year should starts with orientation program to enhance the nurse interns' knowledge and relieve their stress. Nursing faculties are responsible for designing and implementing appropriate internship programs to smooth transition from student to registered nurse. Well designed internship program facilitate interns' entry into clinical practice, support their growth along the continuum of expertise, and development of essential leadership skills within the field of health care ${ }^{(10,11)}$.

Orientation program encourage nurse interns to feel welcome, safe and valued, so it eases transition and provide them with opportunities to develop self confidence, increase job satisfaction and chances to demonstrate competency in the basic skills needed for So this study aimed to assess nurse interns' learning needs before entering clinical practice setting and design a suitable orientation program for covering the gabs in their clinical and management knowledge and skills. 
This study also aimed to evaluate the nurse interns' statistical change of clinical and management knowledge and skills after attending the orientation program.

Aim of the study: This study aimed to apply a designed orientation program for nurse interns based on learning needs assessment through:

- Design, implement, and evaluate an orientation program about clinical and management skills needed for nurse interns based on the findings of using learning needs assessment tool.

\section{Materials and Method:}

Study design: Cross-sectional study design was utilizes to achieve the aim of this study.

Setting: The study was conducted at

Tanta University Faculty of Nursing.

- Tanta University Emergency and Main Hospitals Intensive Care Unites (ICUs) include medical, anesthetic, neurological, neonatal, pediatric, and cardiac .In addition to obstetric and dialysis departments.

providing high quality and safe patient care ${ }^{(5,12,13)}$. Orientation program ensures useful transition time if it is based on the interns' key learning needs and considers teaching and learning activities grounded in practice experience, skilled and well- supported preceptors and structured learning framework $^{(14)}$.

LNAs are often conducted to identify deficiencies in knowledge, skill, behavior, or attitude in the current teaching practices, or to anticipate deficiencies based on expected changes in health care needs. So orientation program based on LNA match the nurse interns' unique learning needs not only nursing service needs ${ }^{(15-18)}$.

\section{Subject}

- All the nursing students (384) passed the fourth academic year (2009-2010) attended the designed orientation program based on their learning needs assessment and participated in pre and post program knowledge test.

All (198) nursing students who spent their internship year (2010-2011) at Tanta University Hospitals were included in post program observation after three months. Tools of the study: to collect the data four tools were used:

1. Learning needs assessment scale, developed by the researcher guided by Zerwekh (2006) and Fakhry (2005) and others, to assess nurse interns' clinical and management learning needs immediately before starting internship year. The sheet contained part of demographic data, and (150) items cover clinical subscales 
(91) items and management subscales (59). Responses were measured by three points Likert Scale for clinical subscale ranging from can do it by myself to can't do it by myself. And two points Likert scale ranging from able to not able for management subscale.

2. Nurse interns' knowledge test, designed by the researcher and used to collect data from nurse interns. The test composed of (100) questions cover both clinical and management subscales. Scoring system represent knowledge levels of nurse interns' ranging from low $(\leq 60 \%)$, medium $(>60 \%-\leq 80 \%)$ to high $(>80)$.

3. Nurse interns' orientation program was designed by the researcher based on the findings of the learning needs assessment scale and nurse interns' knowledge test tools (1\&2) (pre-test). At the end of the program, a post-test was carried out used tool (2) for sample (384) of nurse interns before starting internship year to assess the extent to which desired stated objectives and assumed needs were achieved.

4. Follow up observation sheet, included the same items in the learning needs assessment
5. scale to evaluate nurse interns' actual performance of clinical and management skills after three months of the orientation program during their internship year. Researcher's observations to clinical skills were measured by four points Likert Scale ranged from can do it herself to not applicable. Management skills were measured by two pointsLikert Scale range from able to do to not able to do.

\section{Method of data collection:}

1. An official permission was obtained from responsible authorities including Faculty of Nursing and Hospitals of Tanta University to conduct the study.

2. The learning needs assessment scale was submitted to a jury of ten experts from different nursing specialties to get their suggestions about content validity and applicability of the tool.

3. A pilot study was conducted on ten students out of the proposed sample two times (two weeks separated) to measure test-retest reliability.

4. The content validity index was $(93 \%)$, internal consistency reliability was $(0.9501)$ and the test-retest reliability that was (0.9725). 
5. Nurse interns' learning needs of clinical and management skills were assessed by (tool 1).

6. Nurse interns' knowledge was tested by (tool 2) pre implementation of the orientation program.

7. The nurse interns' orientation program was designed based on the assessment data and literature review. The program was implemented by the researcher and started two weeks just before internship year; it was ten hours duration divided into five sessions each session two hours for five days. The nurse interns' were divided into two groups each group attended the five sessions.

(6\%) graduated from secondary technical nursing school.

About two third $(65 \%)$ of the subject had graduation level very good and (7.6\%) had excellent graduation level. NIs $(71.4 \%)$ did not have previous training. While $(28.6 \%)$ had previous training, $(61.9 \%)$ of them trained during third and fourth academic years, (20.9\%) trained during all the four academic years and (17.2\%) trained during first and second academic years. Training mean period was $(3.4 \pm 0.45)$ month. Those trained NIs $(40.9 \%)$ of them had trained in
8. Nurse interns' knowledge was tested post program by tool (2).

9. Nurse interns' were followed up in their clinical areas for the first three months after the implementation of the orientation program, then they were reevaluated using follow up observation sheet (tool 3).

\section{Results}

Table (1) represents demographic characteristics of nurse interns. Nurse interns $(73.2 \%)$ were in the age group $\leq 20-22$. Majority $(81 \%)$ of nurse_interns graduated from_general secondary school, while minorit

ICUs and $(34.5 \%)$ trained in medical surgical wards.

Figure (1) shows that pre program (51\%) of nurse interns assessed themselves as cannot do, while (49\%) can do both clinical and management skills. More than half (53\%) of NIs assessed themselves as cannot do clinical skills, while (42\%) as cannot do management skills.

Table (2) represents nurse interns' LNA total clinical skills scales pre orientation program. Nurse interns' (71.3\% and 69.1\%) can assess vital signs and collect patient's data respectively. Also (65.5\%, 
$64.5 \%, 64.1 \%, 63.3 \%$ and $60.9 \%$ ) can do assess anthropometric measures, prevent immobility complications, apply safe practice \&infection control, heat\& cold therapy and perform patient hygiene by themselves respectively. Nurse interns $(45.4 \%, 43.7 \%, 42 \%$ and $41.8 \%)$ can not provide by themselves elimination care, assist in diagnostic procedures, provide gastrointestinal care and provide cardio-respiratory care respectively. Around one third (33.1\% and $32.8 \%$ ) of the nurse interns can not provide by themselves newborn care or apply mechanical restraining respectively. More than quarter (29.4\% and 28\%) can not assess by themselves neurological condition or provide wound care respectively.

Table (3) represents nurse interns' LNA total management skills subscales pre orientation program. Nurse interns (56.9\% and 57.9\%) respectively were able to carryout interpersonal and organizational skills. Interpersonal skills showed that nurse interns $(41.3 \%, 41.9 \%$ and $46 \%$ ) were not able to carryout interpersonal relation\& caring, communication, and conflict management skills respectively. Organization skills showed that nurse interns $(44.4 \%, 40.4 \%$ and $41.4 \%$ ) were not able to carryout problem solving, decision making and time management skills respectively. Also (43.6\% and $39.5 \%)$ of interns were not able to carryout priority setting and delegation skills respectively.

Table (4) represents significant difference between nurse interns' total knowledge level on different clinical skills subscales pre\& post orientation program. The table shows statistical significant improvement of NIs' knowledge level post program in all clinical scales at $(\mathrm{P} \leq 0.05)$. High percent $(77 \%)$ of nurse interns' had low and the rest (23\%) had moderate knowledge of clinical skills pre program. Post program NIs (78\%) had high, (20\%) had moderate, while only (2\%) had low knowledge in clinical skills. Pre program NIs (90\%, 74\% and 73\%) had low knowledge of clinical skills on assess comfort\& neurologic state, diagnostic procedures \&collect samples and assess vital signs scales. NIs (61\%, and 58\%) had low knowledge of clinical skills on safe practice\& infection control, gastrointestinal scales respectively. Post program nurse interns (85\%, $84 \%, 84 \%, 74 \%$ and $74 \%$ ) had high knowledge of clinical skills on medication administration\& IV access, collect patient's data, elimination, safe practice\& infection control and newborn care scales respectively. 
Table (5) represents significant difference between nurse interns' total knowledge levels on different management skills subscales pre $\&$ post orientation program. The table shows statistical significant improvement of nurse interns' knowledge level post program in all management scales at $(\mathrm{P} \leq 0.05)$. The majority $(92 \%)$ of the nurse interns' had a low score and the rest $(8 \%)$ of the sample had a moderate score in the management knowledge pre program, while majority (84\%) had a high score and the rest (13\%) had moderate score in the management knowledge post program. Pre program considerable percents $(67 \%, 70 \%, 49 \%$ and $80 \%)$ of interns had low knowledge level on interpersonal, organization, priority setting and delegation skills respectively. Post program, Majority $(81 \%, 85 \%$ and $90 \% 75 \%)$ of the interns had high knowledge level on interpersonal, organization, priority setting and delegation skills respectively.

Table (6) represents correlation between NIs' total clinical knowledge and learning needs assessment subscales of clinical skills pre orientation program. The table reveals a statistical significant positive correlation between the NIs' clinical knowledge and LNA related to safe practice \& infection control $(\mathrm{r}=0.142, \mathrm{P}<0.01)$, cardio-respiratory $\quad(\mathrm{r}=0.152, \quad \mathrm{P}<0.01)$, administration of medication $(\mathrm{r}=0.151, \mathrm{P}<0.01)$ and cold and heat therapy $(\mathrm{r}=0.104, \mathrm{P}<0.05)$.

Table (7) represents correlation between NIs' total management knowledge and learning needs assessment subscales of management skills pre orientation program. The table shows that pre program there was a negative non significant statistical correlation (weak inverse correlation) between the nurse interns' knowledge level and their learning needs assessment related to total interpersonal skills. Although there was a statistical significant positive correlation between the nurse interns' management knowledge and their learning needs assessment related to conflict management skills subscale of interpersonal skills scale $(\mathrm{r}=0.120, \mathrm{P}<0.019)$. 
Table (1). Demographic characteristics of the nurse interns.

\begin{tabular}{|c|c|c|}
\hline \multirow{2}{*}{ Items } & \multicolumn{2}{|c|}{ Nurse Interns $(n=384)$} \\
\hline & no. & $\%$ \\
\hline Age (years) $\leq 20-22$ & 281 & 73.2 \\
\hline$>22$ & 103 & 26.8 \\
\hline Previous graduation school & & \\
\hline General secondary school & 311 & 81.0 \\
\hline Secondary Technical Nursing School & 23 & 6.0 \\
\hline Health Technical Institute & $\mathbf{5 0}$ & $\mathbf{1 3 . 0}$ \\
\hline Graduation level Excellent & 29 & 7.6 \\
\hline Very Good & 250 & 65.1 \\
\hline Good & 78 & 20.3 \\
\hline Satisfactory & 27 & $\overline{7.0}$ \\
\hline Previous training (months) & 274 & 71.4 \\
\hline Yes & 110 & 28.6 \\
\hline First\& second academic years & 19 & 17.2 \\
\hline third \&fourth academic years & 68 & 61.9 \\
\hline All years & 23 & 20.9 \\
\hline $\begin{array}{ll}\text { Training units } & \text { Neonates ICU }\end{array}$ & 13 & 11.8 \\
\hline Medical surgical & 38 & 34.5 \\
\hline Emergency Room & 4 & 3.6 \\
\hline Intensive Care Units & 45 & 40.9 \\
\hline Operation Room & 7 & 6.4 \\
\hline Obstetric & 3 & 2.7 \\
\hline
\end{tabular}




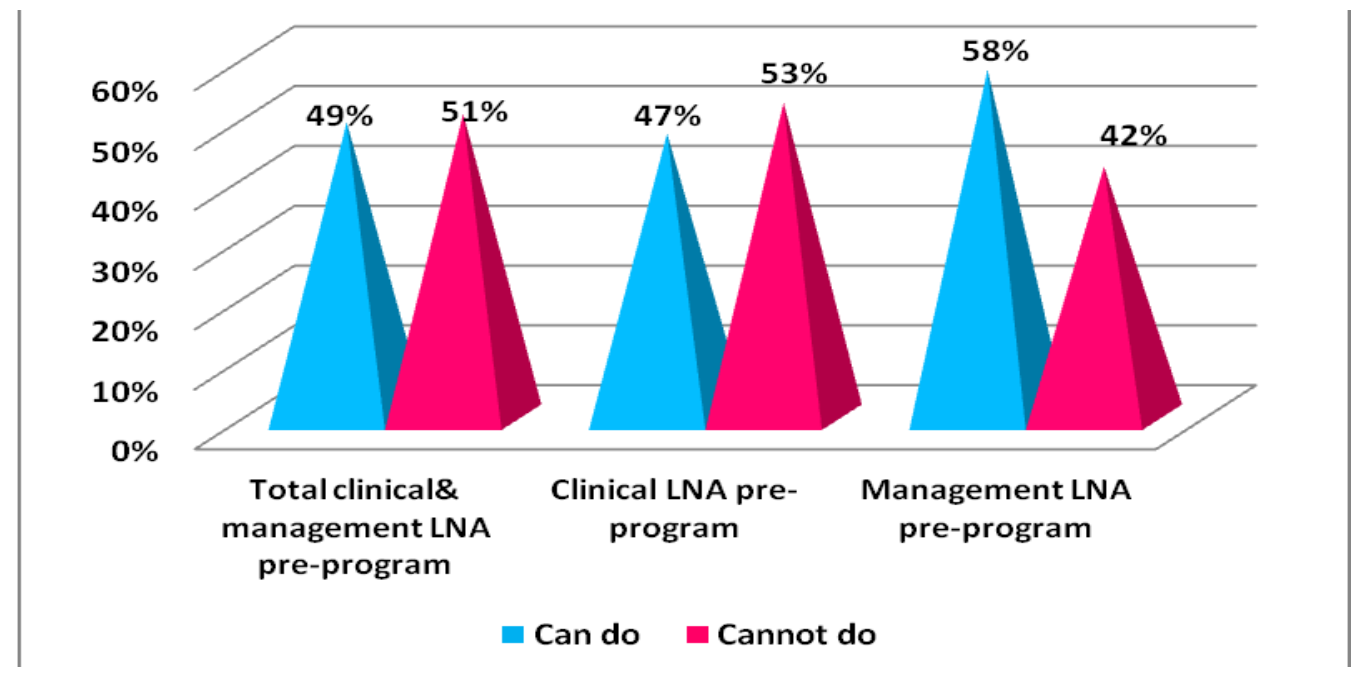

Fig.(1) Nurse interns' learning needs assessment for clinical and management skills pre orientation program. (No.=384).

Table (2). Nurse interns' learning needs assessment total clinical skills scales pre orientation program.

$(\mathrm{No}=384)$.

\begin{tabular}{|c|c|c|c|c|c|c|}
\hline \multirow[t]{2}{*}{ Clinical skills scales } & \multicolumn{2}{|c|}{$\begin{array}{c}\text { Can do it } \\
\text { myself }\end{array}$} & \multicolumn{2}{|c|}{$\begin{array}{c}\text { Can do it } \\
\text { under } \\
\text { supervision }\end{array}$} & \multicolumn{2}{|c|}{\begin{tabular}{|c|} 
Can not do it \\
myself
\end{tabular}} \\
\hline & No & $\%$ & No & $\%$ & No & $\%$ \\
\hline Collect patient's data & 266 & $69.1 \%$ & 83 & $21.4 \%$ & 36 & $9.4 \%$ \\
\hline Assess vital signs & 274 & $71.3 \%$ & 89 & $23.2 \%$ & 21 & $5.5 \%$ \\
\hline Assess anthropometric measures & 252 & $65.5 \%$ & 84 & $21.9 \%$ & 49 & $12.6 \%$ \\
\hline Assess stat of comfort & 187 & $48.6 \%$ & 137 & $35.7 \%$ & 60 & $15.8 \%$ \\
\hline Assess neurological condition & 131 & $34.1 \%$ & 140 & $36.5 \%$ & 113 & $29.4 \%$ \\
\hline Collect samples & 181 & $47.0 \%$ & 104 & $27.0 \%$ & 100 & $26.0 \%$ \\
\hline Assist in diagnostic procedures & 106 & $27.5 \%$ & 111 & $28.8 \%$ & 168 & $43.7 \%$ \\
\hline Safe practice \& infection control & 246 & $64.1 \%$ & 98 & $25.4 \%$ & 40 & 10.5 \\
\hline Provide cardio-respiratory care & 94 & $24.4 \%$ & 130 & $33.8 \%$ & 161 & $41.8 \%$ \\
\hline Provide gastrointestinal care & 89 & $23.1 \%$ & 134 & $34.9 \%$ & 161 & $42.0 \%$ \\
\hline
\end{tabular}




\begin{tabular}{|c|c|c|c|c|c|c|}
\hline Provide elimination care & 100 & $26.1 \%$ & 109 & $28.4 \%$ & 174 & $45.4 \%$ \\
\hline $\begin{array}{l}\text { Medication administration \&IV } \\
\text { access }\end{array}$ & 207 & $\mathbf{5 3 . 8 \%}$ & 86 & $22.3 \%$ & 92 & 23.9 \\
\hline Provide wound care & 159 & $41.3 \%$ & 118 & $30.7 \%$ & 108 & $28.0 \%$ \\
\hline Prevent immobility complications & 248 & $64.5 \%$ & 68 & $17.8 \%$ & 68 & $17.7 \%$ \\
\hline Perform patient hygiene & 234 & $60.9 \%$ & 69 & $18.0 \%$ & 81 & $21.1 \%$ \\
\hline Apply heat \& cold therapy & 243 & $63.3 \%$ & $\overline{70}$ & $18.2 \%$ & 71 & $18.4 \%$ \\
\hline Apply mechanical restraining & 122 & $31.8 \%$ & 136 & $35.4 \%$ & 126 & $32.8 \%$ \\
\hline Provide newborn care & $\overline{143}$ & $37.2 \%$ & $\overline{114}$ & $29.7 \%$ & 127 & $33.1 \%$ \\
\hline
\end{tabular}

Table (3). Nurse interns' learning needs assessment total management skills subscales pre orientation program. $($ No=384).

\begin{tabular}{|c|c|c|c|c|}
\hline \multirow{2}{*}{ Management skills subscales } & \multicolumn{2}{|c|}{ Able } & \multicolumn{2}{|c|}{ Not able } \\
\hline & No & $\overline{\%}$ & No & $\%$ \\
\hline Interpersonal skills & 222 & $56.9 \%$ & 162 & $43.1 \%$ \\
\hline Interpersonal relation \& caring & 225 & $58.7 \%$ & 159 & $41.3 \%$ \\
\hline Communication & 223 & $58.1 \%$ & 161 & $41.9 \%$ \\
\hline Conflict management & 207 & $54.0 \%$ & 177 & $46.0 \%$ \\
\hline Organizational skills & 222 & $57.9 \%$ & 162 & $42.1 \%$ \\
\hline Problem solving & 214 & $55.6 \%$ & 170 & $44.4 \%$ \\
\hline Decision making & 229 & $59.6 \%$ & 155 & $40.4 \%$ \\
\hline Time management & 225 & $58.6 \%$ & 159 & $41.4 \%$ \\
\hline Priority setting & 217 & $56.4 \%$ & 167 & $43.6 \%$ \\
\hline Delegation & 232 & $60.5 \%$ & 152 & $39.5 \%$ \\
\hline
\end{tabular}


Table (4). Significant difference between nurse interns' total knowledge levels on different clinical skills subscales pre\& post orientation program. $($ No.=384)

\begin{tabular}{|c|c|c|c|c|c|c|c|c|}
\hline \multirow{4}{*}{ Clinical } & \multirow{2}{*}{ 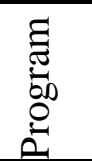 } & \multicolumn{2}{|c|}{ High } & \multicolumn{2}{|c|}{ Moderate } & \multicolumn{2}{|l|}{ Low } & \multirow{2}{*}{$\begin{array}{l}\text { Chi-square } \\
(P \text {-value })\end{array}$} \\
\hline & & No & & No & $\%$ & No & $\%$ & \\
\hline & Pre & 0 & $0 \%$ & 87 & $23 \%$ & 297 & $77 \%$ & \multirow{2}{*}{$0.000 *$} \\
\hline & Post & 299 & $78 \%$ & 76 & $20 \%$ & 9 & $2 \%$ & \\
\hline \multirow{2}{*}{ Collect patient's data } & Pre & 105 & $27 \%$ & 131 & $34 \%$ & 148 & $39 \%$ & \multirow{2}{*}{$0.000 *$} \\
\hline & Post & 321 & $84 \%$ & 40 & $10 \%$ & 23 & $6 \%$ & \\
\hline \multirow{2}{*}{$\begin{array}{l}\text { Assess comfort\& } \\
\text { neurologic state }\end{array}$} & Pre & 5 & $1 \%$ & 35 & $9 \%$ & 344 & $90 \%$ & \multirow{2}{*}{$0.000^{*}$} \\
\hline & Post & 175 & $46 \%$ & 135 & $35 \%$ & 74 & $19 \%$ & \\
\hline \multirow{2}{*}{ Assess vital signs } & Pre & 6 & $2 \%$ & 96 & $25 \%$ & 282 & $73 \%$ & \multirow{2}{*}{$0.000 *$} \\
\hline & Post & 243 & $63 \%$ & 119 & $31 \%$ & 22 & $6 \%$ & \\
\hline \multirow{2}{*}{$\begin{array}{l}\text { Safe practice\& } \\
\text { Infection control }\end{array}$} & Pre & & $11 \%$ & 108 & $28 \%$ & 235 & $61 \%$ & \multirow{2}{*}{$0.000 *$} \\
\hline & Post & 285 & $74 \%$ & 58 & $15 \%$ & 41 & $11 \%$ & \\
\hline \multirow{2}{*}{$\begin{array}{l}\text { Cardio-respiratory } \\
\text { care }\end{array}$} & Pre & & $15 \%$ & 155 & $40 \%$ & 170 & $44 \%$ & \multirow{2}{*}{$0.000 *$} \\
\hline & Post & 320 & $83 \%$ & 56 & $15 \%$ & 8 & $2 \%$ & \\
\hline \multirow{2}{*}{$\begin{array}{l}\text { Diagnostic proc\& } \\
\text { Collect samples }\end{array}$} & Pre & & $4 \%$ & 85 & $22 \%$ & 283 & $74 \%$ & \multirow{2}{*}{$0.000 *$} \\
\hline & Post & 208 & $54 \%$ & 129 & $34 \%$ & 47 & $12 \%$ & \\
\hline \multirow{2}{*}{$\begin{array}{l}\text { Medication admin. } \\
\text { \&IV access }\end{array}$} & Pre & & $16 \%$ & 146 & $38 \%$ & 177 & $46 \%$ & \multirow{2}{*}{$0.000 *$} \\
\hline & Post & 328 & $85 \%$ & 47 & $12 \%$ & 9 & $2 \%$ & \\
\hline \multirow{2}{*}{ Wound care } & Pre & & $15 \%$ & 211 & $55 \%$ & 116 & $30 \%$ & \multirow{2}{*}{$0.000 *$} \\
\hline & Post & 24 & $64 \%$ & 125 & $33 \%$ & 15 & $4 \%$ & \\
\hline \multirow{2}{*}{$\begin{array}{l}\text { Prevent immobility } \\
\text { complication }\end{array}$} & Pre & 29 & $8 \%$ & 84 & $22 \%$ & 271 & $71 \%$ & \multirow{2}{*}{$0.000 *$} \\
\hline & Post & 22 & $58 \%$ & 109 & $28 \%$ & 54 & $14 \%$ & \\
\hline \multirow{2}{*}{ Gastrointestinal care } & Pre & 14 & $4 \%$ & 148 & $39 \%$ & 222 & $58 \%$ & \multirow{2}{*}{$0.000 *$} \\
\hline & Post & 185 & $48 \%$ & 158 & $41 \%$ & 41 & $11 \%$ & \\
\hline
\end{tabular}


Tanta Scientific Nursing Journal

\begin{tabular}{|l|l|ll|ll|ll|l|}
\hline \multirow{2}{*}{ Elimination care } & Pre & 186 & $48 \%$ & 156 & $41 \%$ & 42 & $11 \%$ & \multirow{2}{*}{$0.000^{*}$} \\
\cline { 2 - 8 } & Post & 324 & $84 \%$ & 56 & $15 \%$ & 4 & $1 \%$ & \\
\hline \multirow{2}{*}{ Patient hygiene } & Pre & 69 & $18 \%$ & 197 & $51 \%$ & 118 & $31 \%$ & \multirow{2}{*}{$0.000^{*}$} \\
\cline { 2 - 8 } & Post & 258 & $67 \%$ & 112 & $29 \%$ & 14 & $4 \%$ & \\
\hline \multirow{2}{*}{ Restrain } & Pre & 67 & $17 \%$ & 176 & $46 \%$ & 141 & $37 \%$ & \multirow{2}{*}{$0.000^{*}$} \\
\cline { 2 - 7 } & Post & 254 & $66 \%$ & 103 & $27 \%$ & 27 & $7 \%$ & \\
\hline \multirow{2}{*}{ Newborn care } & Pre & 61 & $16 \%$ & 221 & $58 \%$ & 102 & $27 \%$ & \multirow{2}{*}{$0.000^{*}$} \\
\cline { 2 - 7 } & Post & 284 & $74 \%$ & 83 & $22 \%$ & 17 & $4 \%$ & \\
\hline
\end{tabular}

*significant at $p \leq 0.05$ 
Table (5). Significant difference between nurse interns' total knowledge levels on different management skills subscales pre\& post orientation program. (No.=384)

\begin{tabular}{|c|c|c|c|c|c|c|c|c|}
\hline \multirow{4}{*}{ Total nowledge } & \multirow{2}{*}{$\begin{array}{l}E \\
\vdots \\
0 \\
0 \\
0\end{array}$} & \multicolumn{2}{|c|}{ High } & \multicolumn{2}{|c|}{ Moderate } & \multicolumn{2}{|l|}{ Low } & \multirow{2}{*}{$\begin{array}{l}\mathrm{X}^{2} \\
(P \text {-value })\end{array}$} \\
\hline & & No & $\%$ & No & $\%$ & No & $\%$ & \\
\hline & Pre & 0 & $0 \%$ & 32 & $8 \%$ & 352 & $92 \%$ & \multirow{2}{*}{$0.000 *$} \\
\hline & Post & 323 & $84 \%$ & 48 & $13 \%$ & 13 & $3 \%$ & \\
\hline \multirow{2}{*}{ Interpersonal Skills } & Pre & 21 & $6 \%$ & 104 & $27 \%$ & 259 & $67 \%$ & \multirow{2}{*}{$0.000 *$} \\
\hline & Post & 293 & $81 \%$ & 74 & $16 \%$ & 17 & $3 \%$ & \\
\hline \multirow{2}{*}{$\begin{array}{l}\text { Interpersonal relation \& } \\
\text { caring }\end{array}$} & Pre & 16 & $4 \%$ & 116 & $30 \%$ & 252 & $66 \%$ & \multirow{2}{*}{$0.000 *$} \\
\hline & Post & 280 & $73 \%$ & 91 & $24 \%$ & 13 & $3 \%$ & \\
\hline \multirow{2}{*}{ Communication } & Pre & 42 & $11 \%$ & 141 & $37 \%$ & 201 & $52 \%$ & \multirow{2}{*}{$0.000 *$} \\
\hline & Post & 315 & $82 \%$ & 57 & $15 \%$ & 12 & $3 \%$ & \\
\hline \multirow{2}{*}{ Conflict management } & Pre & 6 & $2 \%$ & 53 & $14 \%$ & 325 & $85 \%$ & \multirow{2}{*}{$0.000 *$} \\
\hline & Post & 286 & $74 \%$ & 74 & $19 \%$ & 24 & $6 \%$ & \\
\hline \multirow{2}{*}{ Organization skills } & Pre & 149 & $12 \%$ & 99 & $18 \%$ & 235 & $70 \%$ & \multirow{2}{*}{$0.000 *$} \\
\hline & Post & 291 & $85 \%$ & 52 & $5 \%$ & 41 & $10 \%$ & \\
\hline \multirow{2}{*}{ Time management } & Pre & 52 & $14 \%$ & 172 & $45 \%$ & 160 & $42 \%$ & \multirow{2}{*}{$0.000 *$} \\
\hline & Post & 279 & $73 \%$ & 89 & $23 \%$ & 16 & $4 \%$ & \\
\hline \multirow{2}{*}{ Problem solving } & Pre & 81 & $21 \%$ & 0 & $0 \%$ & 303 & $79 \%$ & \multirow{2}{*}{$0.000 *$} \\
\hline & Post & 300 & $78 \%$ & 0 & $0 \%$ & 84 & $22 \%$ & \\
\hline \multirow{2}{*}{ Decision making } & Pre & 16 & $4 \%$ & 125 & $33 \%$ & 243 & $63 \%$ & \multirow{2}{*}{$0.000^{*}$} \\
\hline & Post & 293 & $76 \%$ & 67 & $17 \%$ & 24 & $6 \%$ & \\
\hline \multirow{2}{*}{ Priority setting skills } & Pre & 78 & $20 \%$ & 116 & $30 \%$ & 190 & $49 \%$ & \multirow{2}{*}{$0.000 *$} \\
\hline & Post & 346 & $90 \%$ & 32 & $8 \%$ & 6 & $2 \%$ & \\
\hline \multirow{2}{*}{ Delegation skills } & Pre & 1 & $0 \%$ & 76 & $20 \%$ & 307 & $80 \%$ & \multirow{2}{*}{$0.000 *$} \\
\hline & Post & 288 & $75 \%$ & 68 & $18 \%$ & 28 & $7 \%$ & \\
\hline
\end{tabular}

*significant at $p \leq 0.05$ 


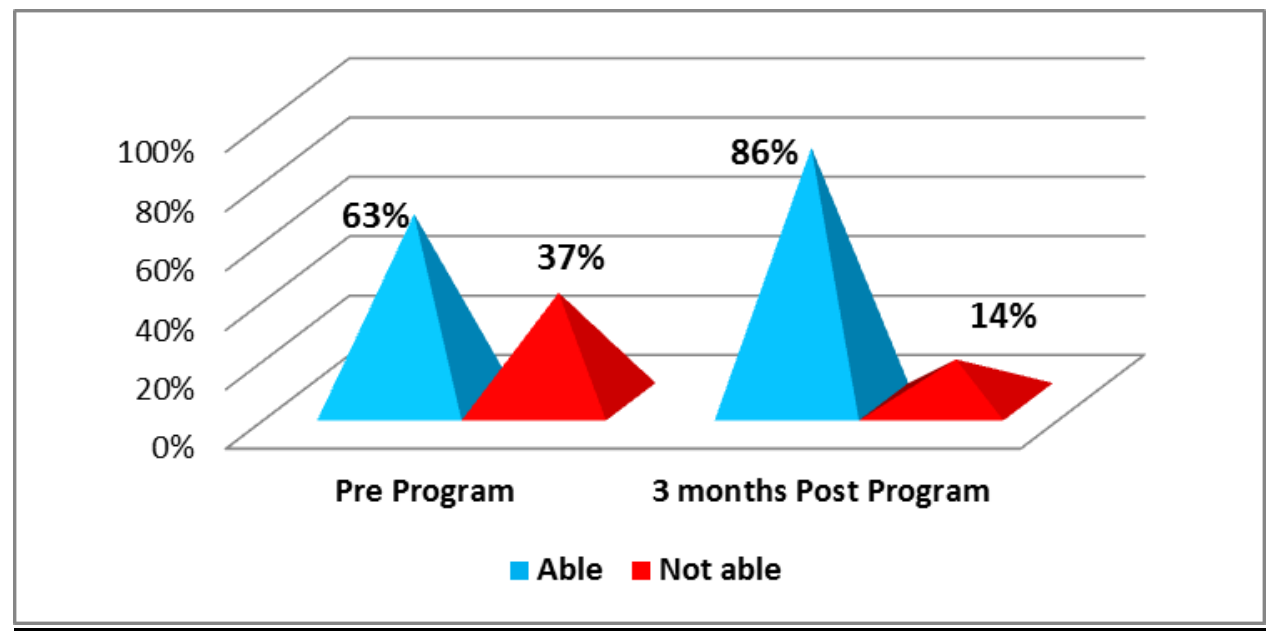

Fig.(2): Nurse interns' pre orientation program learning needs assessment (for both total management and clinical skills) and researcher's observation3 months post program. (No.=198)

Table (6). Correlation between nurse interns' total clinical knowledge and learning needs assessment subscales of clinical skills pre orientation program. $($ No. $=384)$.

\begin{tabular}{|l|c|c|}
\hline \multirow{2}{*}{$\begin{array}{l}\text { Clinical knowledge and learning } \\
\text { needs assessment subscales. }\end{array}$} & \multicolumn{2}{|c|}{$\begin{array}{c}\text { Correlation between knowledge \& } \\
\text { learning needs assessment pre program }\end{array}$} \\
\cline { 2 - 3 } & $\mathrm{r}$ & $P$-value \\
\hline Collect patient's data & 0.070 & 0.172 \\
\hline Assess comfort\& neurologic state & -0.039 & 0.451 \\
\hline Assess vital signs & 0.007 & 0.884 \\
\hline Safe practice\& Infection control & $0.142^{* *}$ & 0.005 \\
\hline Cardio-respiratory care & $0.152^{* *}$ & 0.003 \\
\hline Diagnostic pros\& Collect samples & 0.053 & 0.300 \\
\hline Medication administration \&IV & & 0.003 \\
access & $0.151^{* *}$ & 0.056 \\
\hline Wound care & 0.097 & \\
\hline
\end{tabular}




\begin{tabular}{|l|c|c|}
\hline Prevent immobility complications & 0.070 & 0.173 \\
\hline Gastrointestinal care & 0.048 & 0.353 \\
\hline Elimination care & 0.008 & 0.882 \\
\hline Patient hygiene & 0.065 & 0.207 \\
\hline Cold and heat therapy & $0.104^{*}$ & 0.041 \\
\hline Applying mechanical restrain & -0.058 & 0.255 \\
\hline Newborn care & 0.092 & 0.073 \\
\hline
\end{tabular}

*. Correlation is significant at the 0.05 level. **. Correlation is significant at the 0.01 level.

Table (7). Correlation between nurse interns' management total knowledge and learning needs assessment subscales of management skills pre orientation program. (No.= 384)

\begin{tabular}{|c|c|c|}
\hline \multirow{2}{*}{$\begin{array}{l}\text { Management knowledge and } \\
\text { learning needs assessment } \\
\text { subscales }\end{array}$} & \multicolumn{2}{|c|}{$\begin{array}{l}\text { Correlation between knowledge \& } \\
\text { learning needs assessment pre program }\end{array}$} \\
\hline & $\mathrm{r}$ & $P$-value \\
\hline Interpersonal skills & -0.024 & 0.637 \\
\hline $\begin{array}{l}\text { Interpersonal relation \& } \\
\text { caring }\end{array}$ & 0.033 & 0.524 \\
\hline Communication & -0.045 & 0.377 \\
\hline Conflict management & $0.120 *$ & 0.019 \\
\hline Organizational skills & 0.089 & 0.081 \\
\hline Problem solving & 0.089 & 0.081 \\
\hline Decision making & 0.065 & 0.206 \\
\hline Time management & 0.041 & 0.421 \\
\hline Priority setting & 0.038 & 0.453 \\
\hline Delegation & 0.002 & 0.977 \\
\hline
\end{tabular}

*Correlation is significant at the 0.05 level . 


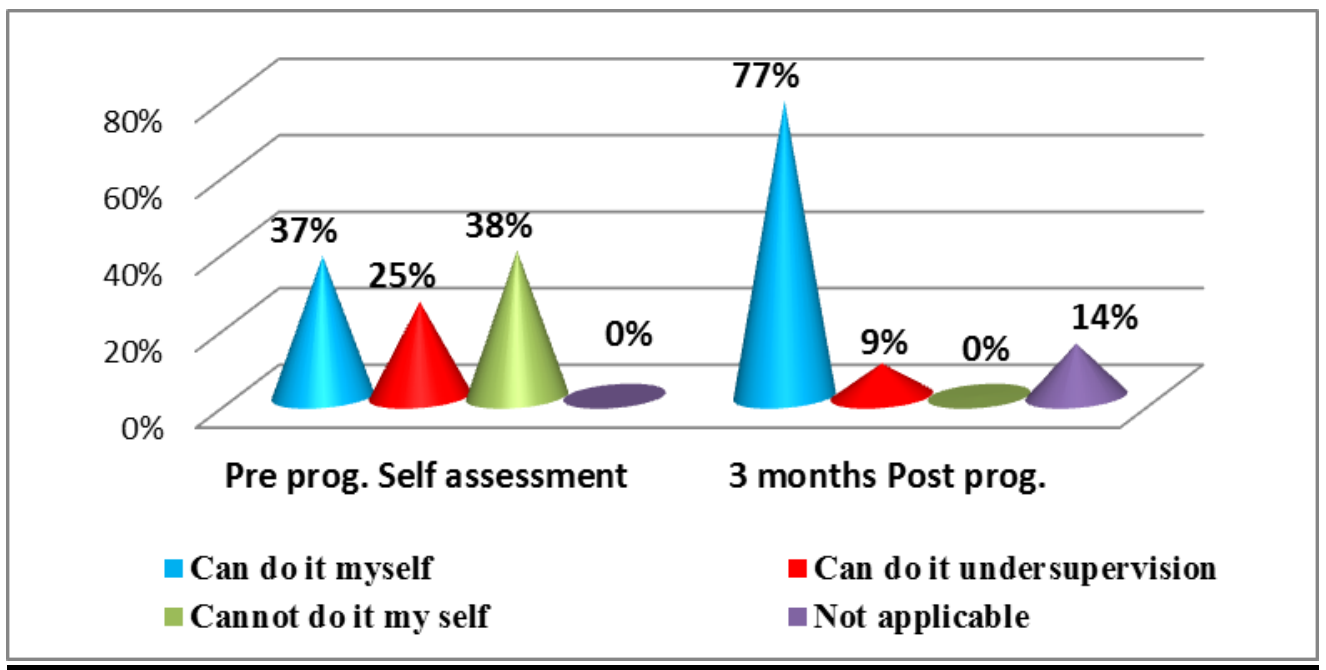

Fig.(3) Nurse interns' LNA of total clinical skills pre program and researcher's observation3 months post orientation program. $($ No.=198)

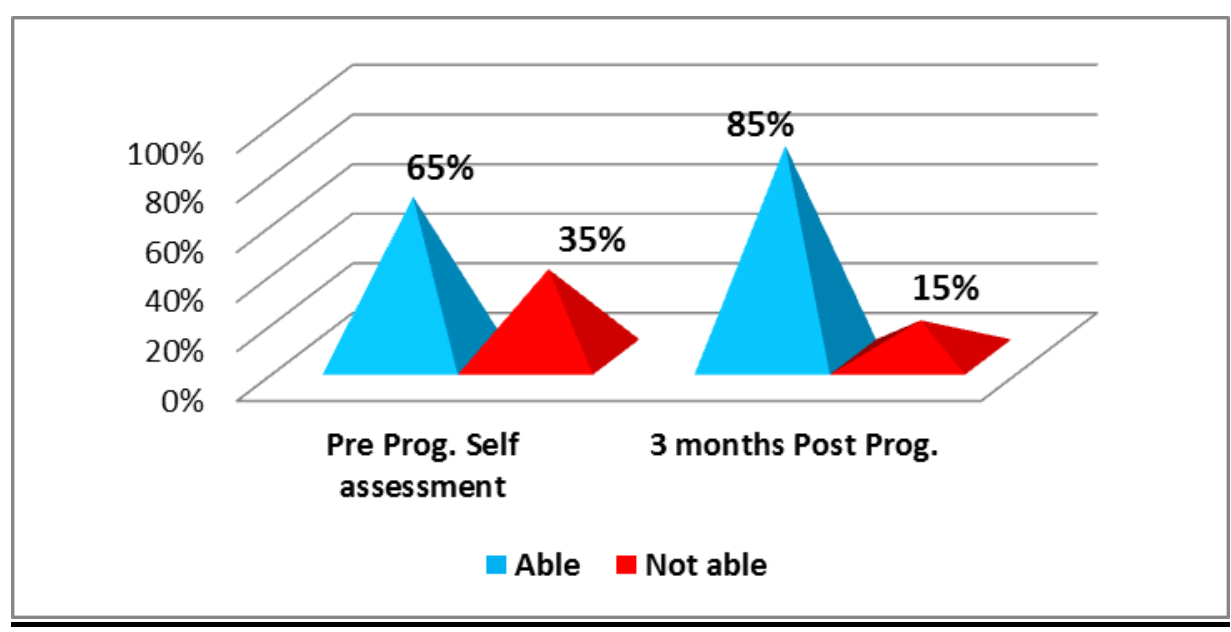

Fig.(3) Nurse interns' LNA of total clinical skills pre program and researcher's observation3 months post orientation program. $($ No.=198) 


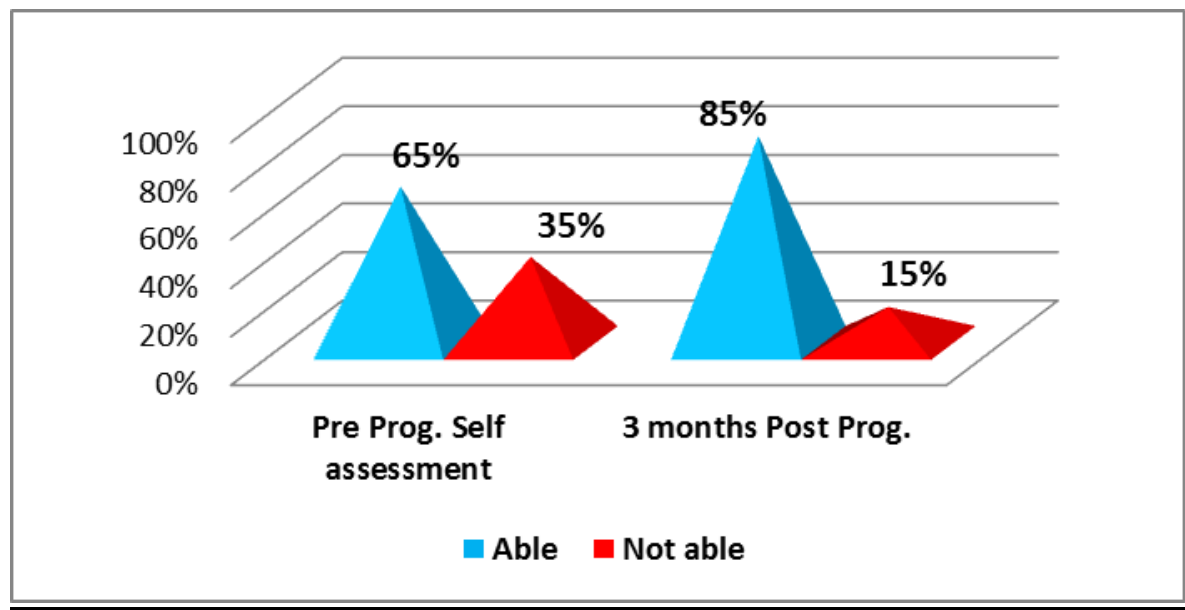

Fig.(4) Nurse interns' learning needs assessment pre orientation program and researcher's observation3 months post program of total management skills. (No.=198)

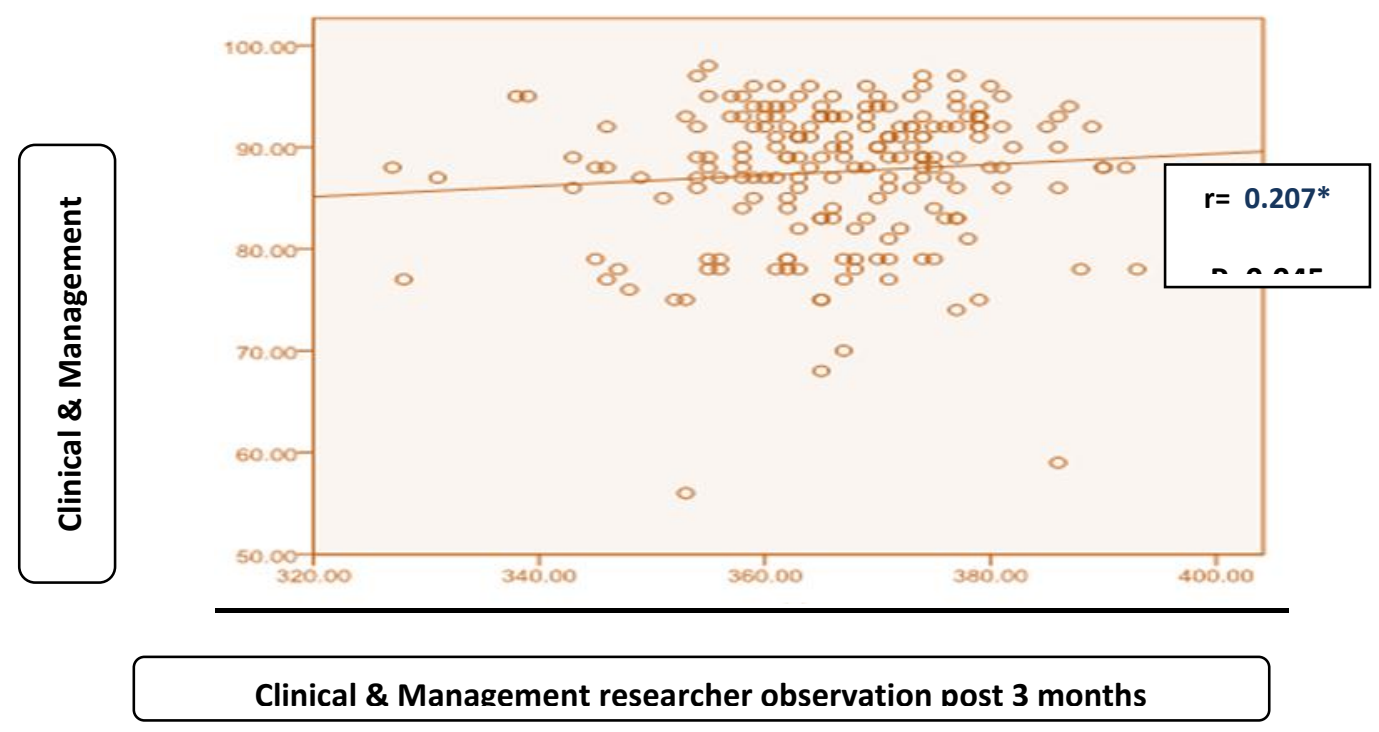

Fig. (5) Correlation between nurse interns' total knowledge (clinical and management) and researcher's observation 3 months post program. 


\section{Discussion}

Learning needs assessment guarantee a relevant and accurate pre-internship orientation program thus fosters a smooth transition from nursing students to professional registered nurse. Results of using a well designed learning needs assessment (LNA) tools can guide nursing faculties to fill in the theory practice gap in NIs' performance and strengths the weak points in the nurse interns' knowledge.

The present study results revealed no statistical significant correlation between NIs' learning needs self assessment and their pre-orientation program knowledge on clinical and management skills. Around half of NIs assessed themselves as can do clinical and management skills; in despite of the incorrect answers for the majority of NIs on basic knowledge test questions about clinical and management skills. Most probably this contradiction is due to their overestimation of their abilities. So those NIs require continuous and constructive feedback on their abilities and progress to identify gaps between their practice and related basic knowledge. Khamis (2009) study about the impact of management program on competencies of NGs during internship year supported results of the

present study and found a remarkable disparity between nurse interns' knowledge and the level of their practical skills ${ }^{(19)}$.

Feedback is an effective method of improving performance because it allows nurse interns to correct earlier misunderstandings that developed during learning process. Providing feedback can be done fairly quickly, with little expenditure of time to improve the learning process in clinical setting and ensure interns' master of clinical tasks.

The results of present study revealed that there is a statistical significant correlation between nurse interns' total knowledge (clinical and management) with their previous training, their graduation level and their previous graduation school. Really NIs got high graduation level and had previous training in private hospitals specially those trained in ICUs as well as those graduated from institute or nursing schools got opportunities to apply their learned skills in 
practical situations, helped them to interact with more experienced healthcare staff and improved their skills performance. Beccroft (2009) study about "internship outcomes of one year pilot program" supported the present study findings, and found that high percent of nursing interns whom had an excellent graduation level showed high competency level, while only low percent of nursing interns who have a good graduation level showed moderate level of competency $^{(1)}$.

Findings of present study showed that more than half of NIs assessed themselves as cannot do both clinical standard and management skills. Those NIs' clinical knowledge test preprogram showed that they had poor knowledge level regarding both clinical and management skills.

The fact is that those NIs' knowledge were poor about ten out of eighteen skills understudy including assess state of comfort, assess neurological condition, collect samples, assist in diagnostic procedures, apply safe practice and infection control measures and apply mechanical restrain. Besides, they had poor knowledge level regarding cardio-respiratory, gastrointestinal, elimination, wound, and newborn care skills.

The results of LNA revealed that around forty percent of nurse interns assessed themselves as being not able to carryout all the management skills. Those NIs were not able to carryout interpersonal skills, organizational skills, priority setting and delegation skills. Majority of those interns showed low level of management knowledge and minority were at moderate level of knowledge.

These results may be because at the beginning of their transition NIs suspect their management knowledge and skills, they still not have any refreshing courses or orientation program. Nurse interns look for internship years as the sparkling beginning of their professional carrier, and they will be treated as a trainee, they dream with practicing under supervision of highly experienced nursing staff, whom will provide them with direction. Those interns need specific job description that clarify their role and are in need of pre-internship 
orientation program to support their skills and refresh their knowledge.

The present study findings confirmed by Morrow (2009) whom found that novice nurses were inadequately prepared in the areas of critical thinking skills, decision making and problem solving ${ }^{(20)}$. Also Greenwood (2000) reported that novice nurses had poor time management skills and prioritization, and a general inability to convert theoretical training to real-world situations ${ }^{(21)}$.

Those NIs were not able to do interpersonal relation\& caring, communication and conflict management skills. Their lack of interpersonal management skills knowledge is the apparent cause for their inability to carryout these skills. Contradicting to the present study findings Arthur (2008) studied assessing nursing students' basic communication and interviewing skills found that nurse interns rated themselves as able to provide good therapeutic interpersonal relations and communication skills while providing practical care ${ }^{(22)}$. Present study LNA revealed that nurse interns assessed themselves as being not able to carryout conflict management skills. This can be interpreted as the pre-graduate education did not sufficiently prepare those interns for managing conflict. They studied lectures on conflict resolution; but they did not have enough chance to apply theory into practice. Even during their undergraduate practice in healthcare setting the instructors try to overly protect their students and resolve any conflict with patients, nurses, doctors, or even with their colleagues. Obied (2008) study about management of workplace violence against nurses confirmed present study and found that NIs' lack of conflict resolution strategies and interpersonal skills constitute one of the main reasons behind NIs' experience of workplace violence ${ }^{(23)}$.

The results of the LNA of present study showed that nurse interns assessed themselves as being not able to carryout organizational skill. They were not able to do each of solve problems, make decisions, or even mange their time. Those interns showed low knowledge regarding organizational skills. Those NIs lacking of organizational knowledge and skills make 
them to feel inadequate and increase their fear of harming patients or miss any care. They have to learn how to group simultaneous interventions to master their own time, skills, and be able to provide care independently with no external guidance. So those NIs require orientation program on management organization knowledge to support them to regain self confidence.

Based on the findings of LNA of present study the nurse interns assessed themselves as being not able to carryout interpersonal skills.

Findings of LNA illustrated that nurse interns assessed themselves as being not able to carryout decision making skills. Most probably this result reflects their lack of decision making skills knowledge. They answer incorrectly about questions related to timing of decision making, steps of decision making process and personal barriers of decision making. So it is suggested that a formal orientation program that enhance critical thinking and decision making ability should be given to NIs to improve their knowledge and skills preinternship year. Consequently they will be clinically effective in making decisions and reduce the risk of committing errors.

Gillespie \& Peterson (2009) study about helping novice nurses make effective clinical decisions supported the present study findings and reported that many novice nurses fall in different errors as they lack critical thinking and decision making skills. They stated that novice nurses with limited experience in the care settings, in which they work, tend to view decisionmaking as nearly responding to patient complaints and following protocols or documented care plans. As they make decisions, their focus leans toward doing, rather than on thinking and reflecting ${ }^{(24)}$.

LNA findings of present study revealed that nurse interns assessed themselves as being not able to carryout time management skills. Those interns were not able to manage distractions, use time saving strategies, avoid doing others' work, or even refuse responsibility they cannot manage. They cannot finish most of things they start or being sensitive for others time. The fact is that those NIs' lack knowledge about each of how to manage their time, prioritize 
competing tasks in a way that best serves patients needs, respects the team, and maintains energy and focus throughout the shift. So they need pre-orientation program include educational materials on prober time management.

Smith \& Crawford (2003) supported present study results and found that novice nurses is delayed in patient care, due to their failure to administer in a timely manner, failure to recognize and failure to intervene in relation to patient care ${ }^{(25)}$.

However most NGs lack the judgment, confidence, and experience to act decisively, until they fully develop their clinical judgment, they must follow the rules. If the rules don't cover a particular situation, they must rely on more experienced team members for advice and support. Further confusion may arise when new nurses are called to help teammates while operating within the multiple time demands of a shift.

Saintsing et al. (2011) study about novice nurse's clinical decision-making and how to avoid errors support the present study results and found that the issues of time management and critical thinking are two items that are potentially dependent upon each other. Novice nurse is likely to make judgment errors regarding critical thinking related to real or perceived time constraints $^{(26)}$.

LNA results showed that NIs assessed themselves as being not able to carry out delegation skills. Those NIs do not trust others to carryout their duties and do not have the courage to take responsibility for their delegated skills. They cannot select and organize tasks to be delegated, cannot select appropriate person for delegation, or even maintain reasonable control for delegated tasks. Those NIs got low level of knowledge regarding delegation skills. Ruff (2011) study about delegation skills: essential to the contemporary nurse supported the present study and found that ineffective delegation was cited by nursing staff as one reason for missed care ${ }^{(27)}$.

LNA revealed that NIs assessed themselves as being not able to carry out priority setting skills, which matches their low level of knowledge regarding priority setting. Those nurse interns cannot write pros and cons for 
options or priorities tasks according to goals. In addition, they cannot do each of put clear goals and objectives, organize their thoughts or even take time to plan their activities. Most probably those NIs also lack experience of early detection and intervention for priority cases which nursing care can prevent its deterioration.

Immediate post implementation of the present study well design pre-internship orientation educational program, NIs changed significantly from being with low to be with high level of knowledge on clinical skills standards procedures; related to collect patient's data, cardio-respiratory care, medication administration, elimination care, prevent immobility complications, wound care, diagnostic procedures and safe practice and infection control; statistically significantly improved post orientation program. As well as, an improvement in management knowledge level showed for interpersonal, organizational, priority setting and delegation skills. But still some NIs had low knowledge level regarding clinical and management skills, whom need to study their learned materials.
The findings of the present study is also supported by Roussel et al. (2006) study on management and leadership for nurse administrators asserted that educational program helped nurses in keeping up to data with new concepts, increasing knowledge and competences, modifying their attitudes and developing their abilities to deal with patients and problems ${ }^{(28)}$.

The present study researcher's followed up NIs during their first three months of internship year to direct them for proper skill acquisition and socialization. The researcher arranged that NIs attend simple orientation at the first three morning shifts at the beginning of each rotation, to help them to be familiar with the unit environment, procedures and staff this was carried out in cooperation with the $\mathrm{HN}$ of each unit. Each NI was assigned to care for one patient with experienced $\mathrm{RN}$ for first week. The TUH HNs and number of RNs were invited to attend with NIs their the preinternship orientation program implemented by the researcher, to be sure for refreshing their knowledge and to facilitate the future interns' socialization and support. 
The researcher intended to help NIs socialization process by which they will acquire the specialized knowledge, skills, values, norms and interests needed to perform their roles acceptably. Beside the facilitation for professional socialization process that integrates the cognitive and affective domains of professional performance governs their behavior. The researcher believes the importance that learning process takes place in a social environment, so that the learning occurs through interaction with their actual work units. Really the attention and the clinical supervision which NIs received at the follow up period of internship orientation program enhanced their sense of confidence and improve their competence. As well as improved their performance to role, responsibilities and patients care quality.

Data analysis of follow up observation sheet three months post orientation program revealed significant improvement of NIs' ability to carryout all clinical skills, in comparison with their learning needs self assessment pre-orientation program. Researcher's observation also revealed that majority of nurse interns can by themselves assess vital signs, state of comfort, collect samples, provide cardio-respiratory care, apply mechanical restrain and provide newborn care. As well as NIs can by themselves prevent immobility complications, provide wound care, assess neurological condition and carryout safe practice and infection control measures.

According to the researcher's observation three months post orientation program nurse interns' ability to carryout management skills of interpersonal, organizational, priority setting and delegation skills were significantly improved, in comparison with their learning needs self assessment preorientation program. So nurse interns should attend immediate pre-internship orientation programs to help them to refresh their knowledge, memorize the basic clinical and management standards needed to be applied during their internship year. Learning needs assessment is very important to meet nurse interns' individual needs and to address the skills required for specific practice areas. Beside their knowledge must be tested to identify their actual educational needs. 
Designing, implementing the orientation program and using different teaching methods helped to evoke nurse interns maximum benefits and to overcome their individual differences. Really present experience assured that at the beginning of the internship year NIs need to be supported, encouraged and mentored by the faculty staff members until they became confident in their clinical and management practice

\section{References}

1. Beecroft P.C., Kunzman L., and Krozek Ch. RN Internship: Outcomes of a One-Year Pilot Program. Journal of Nursing Administration. 2001; 31(12):575-82.

2. El-Sayed S.H., Zakaria A.M. and Shazely M.M. Management Function and Managerial Skills for Nurse Interns at Mansoura University Hospitals. The New Egyptian Journal of Medicine.2007; 36(5):14-22.

3. Cantrell M. and Browne A. The impact of a nurse externship program on the transition process from graduate to registered nurse, Journal for Nurses in
Staff Development.2005; 21 (6) : 249256.

4. AboRamadan A. H. Job Analysis and Description for Nursing Interns at Tanta University Hospitals. Master thesis. Faculty of nursing. Tanta University. 2006.

5. Association of Registered Nurses of Newfoundland and Labrador. Orientation Programs for Registered Nurses: Best Practice Guidelines. (2003). ARNNL .Council Policy Ends, St. John's, NL: Author.

6. Casey K., Fink R., Krugman M. and Propst J. The graduate nurse experience. Journal of Nursing Administration. 2004; 34 (6), 303-311.

7. Oermann M.H. and Garvin M.F. Stresses and challenges for new graduates in hospitals. Nurse Educator Today. 2002; 22: 225-230.

8. Butler K.M. and Hardin-Pierce M. Leadership strategies to enhance the transition from nursing student role to professional nurse. Nursing Leadership Forum. 2005; 9 (3), 110-117. 
9. Grant J. Learning needs assessment: assessing the need. BMJ. 2002; 324:1569.

10. Lenburg C. Vermot Nurse Internship Project, retrieved from http//www.nursingworld.org/ojin/topic10 /tpc.htm. (2000).

11. El-Shimy H.M. Effect of Implementing an Orientation Program On The Nurse Interns. Egyptian Medical Journal. 1996; 13(1):25-31.

12. Stout D. "Performance Analysis for Training". Niagara Paper Company. 1995. Niagara, WI.

13. Kelly P. Nursing Leadership\& Management. $3^{\text {rd }}$ ed. Delmar, Cengage Learning.USA. 2012:674.

\section{Adlam K. -A ., Dotchin M. \&}

Hayward S. Nursing first year of practice, past, present and future: documenting the journey in New Zealand. Journal of Nursing Management; 2009; 17: 570-575.

15. Hassan R.M. The performance of Nursing Interns Related to Basic Nursing Procedures. Unpublished thesis, Faculty of Ain Shams University.(2001):101-2
16. Onsy N. Impact of Internship Training Experience on Nurses' Interns skill Acquisition, Unpublished thesis, Faculty of Ain Shams University.1998: 122-3

17. Ratnapalan S. and Hilliard R.I. Needs Assessment in Postgraduate Medical Education: A Review. Med Educ Online [serial online] 2002;7:8. Available from URL http://www.med-edonline.org

18. Donald E.M.J. and, Donald L.C. Needs assessment. In: Continuing Medical Education: A Primer.Westport: CT: Praeger, 1992.

19. Khamis L. Impact of Management Program on Competencies of Baccalaureate Nursing Graduates During Internship Year. Unpublished Unpublished Doctoral Thesis. Faculty of Nursing. Tanta University. 2009.

20. Morrow S. New graduate transitions: leaving the nest, joining the flight. Journal of Nursing Management. 2009; 17: 278- 287.

21. Greenwood J. Critique of the graduate nurse: an international 
perspective. Nurse Education Today. 2000; 20: 17-23.

22. Arthur D. Assessing nursing students' basic communication and interviewing skills: the development and testing of a rating scale. Journal of Advanced Nursing. 2008; 29(3):658-659.

23. Obied H.K. Management of workplace violence in Emergency Hospital Tanata University.2008. Unpublished Master thesis. Faculty of Nursing Tanta University.

\section{Gillespie M. and Paterson B. L.} Helping Novice Nurses Make Effective Clinical Decisions: The Situated Clinical Decision-Making Framework. Nursing Education Perspectives. 2009; 30 (3):164-170.

\section{Smith J. \& Crawford L.} Medication errors and difficulty in first patient assignments of newly licensed nurses. JONAS Healthcare Law, Ethics and Regulation. 2003; 5: 65-67.

26. Saintsing D. , GIBSON L .M. and Pennington A.W. The novice nurse and clinical decision-making: how to avoid errors. Journal of Nursing Management. 2011; 19:354-359.

27. Ruff V. A. "Delegation Skills: Essential to the Contemporary Nurse" (2011). Master of Arts in Nursing Theses. St. Catherine University Paper 21.

http://sophia.stkate.edu/ma_nursing/21

28. Roussel L. and Swansburage R C. Management and Leadership for nurse administrators. $4^{\text {th }}$ ed., Boston, Jones and Barlett Publ. Inc. 2006: 45,47-8, 